\title{
CLIMP-63 is a gentamicin-binding protein that is involved in drug-induced cytotoxicity
}

\author{
T Karasawa ${ }^{\star, 1}$, Q Wang ${ }^{1}$, LL David ${ }^{2}$ and PS Steyger ${ }^{1}$
}

Aminoglycoside-induced nephrotoxicity and ototoxicity is a major clinical problem. To understand how aminoglycosides, including gentamicin, induce cytotoxicity in the kidney proximal tubule and the inner ear, we identified gentamicin-binding proteins (GBPs) from mouse kidney cells by pulling down GBPs with gentamicin-agarose conjugates and mass spectrometric analysis. Among several GBPs specific to kidney proximal tubule cells, cytoskeleton-linking membrane protein of $63 \mathrm{kDa}$ (CLIMP-63) was the only protein localized in the endoplasmic reticulum, and was co-localized with gentamicin-Texas Red (GTTR) conjugate after cells were treated with GTTR for $1 \mathrm{~h}$. In western blots, kidney proximal tubule cells and cochlear cells, but not kidney distal tubule cells, exhibited a dithiothreitol (DTT)-resistant dimer band of CLIMP-63. Gentamicin treatment increased the presence of DTT-resistant CLIMP-63 dimers in both kidney proximal (KPT11) and distal (KDT3) tubule cells. Transfection of wildtype and mutant CLIMP-63 into 293T cells showed that the gentamicin-dependent dimerization requires CLIMP-63 palmitoylation. CLIMP-63 siRNA transfection enhanced cellular resistance to gentamicin-induced toxicity, which involves apoptosis, in KPT11 cells. Thus, the dimerization of CLIMP-63 is likely an early step in aminoglycoside-induced cytotoxicity in the kidney and cochlea. Gentamicin also enhanced the binding between CLIMP-63 and 14-3-3 proteins, and we also identified that 14-3-3 proteins are involved in gentamicin-induced cytotoxicity, likely by binding to CLIMP-63.

Cell Death and Disease (2010) 1, e102; doi:10.1038/cddis.2010.80; published online 18 November 2010

Subject Category: Experimental Medicine

Aminoglycosides are among the most commonly used antibiotics in clinical practice, ${ }^{1}$ and are essential for treating life-threatening tuberculosis and Gram-negative bacterial infections, such as meningitis, pulmonary infections in cystic fibrosis patients, and bacterial sepsis in infants. ${ }^{2-4}$ However, aminoglycosides can induce severe acute nephrotoxic and permanent ototoxic side effects. Following systemic administration, aminoglycosides are taken up by, and are cytotoxic to, kidney proximal tubule epithelial cells, the primary site of nephrotoxicity. Renal toxicity is largely reversible because of the regenerative tubular epithelial cell proliferation. ${ }^{5}$ Aminoglycoside-induced ototoxicity, on the other hand, is mostly permanent because these drugs primarily induce cytotoxicity in cochlear sensory hair cells, which cannot spontaneously regenerate in mammals following hair cell death. ${ }^{6}$

Aminoglycosides enter eukaryotic cells by endocytosis and are trafficked to lysosomes, the Golgi complex and endoplasmic reticulum (ER) before being released into the cytosol. $^{7-9}$ Aminoglycosides also permeate non-selective cation channel directly into the cytoplasm. ${ }^{10-12}$ In the cell, aminoglycosides can induce cytotoxicity that results in both apoptotic and non-apoptotic cell death by activating caspase-3, caspase-9, c-jun N-terminal kinases, calpains and cathespin $\mathrm{D}$, or generating toxic levels of reactive oxygen species. ${ }^{13-17}$
How aminoglycosides induce these cell death mechanisms remains unclear.

In this study, we identified gentamicin-binding proteins (GBPs) from proximal tubule cells that may contribute to drug susceptibility in these cells. Of these proteins, only the cytoskeleton-linking membrane protein of $63 \mathrm{kDa}$ (CLIMP-63) is localized in the ER membrane, and colocalized with gentamicin-Texas Red (GTTR). CLIMP-63, also known as CKAP4, is a type II transmembrane protein with a short cytosolic domain and a long lumenal domain. The physiological functions of CLIMP-63 have not been well documented, but it is thought to be an anchoring protein connecting the ER to the cytoskeleton. ${ }^{18}$ The cytosolic domain of CLIMP-63 anchors the ER network to the microtubular cytoskeleton, and the lumenal domain of CLIMP-63 is required for its oligomerization and immobilization in the ER membrane. ${ }^{18,19}$ We show that gentamicin binds to the lumenal domain and induces dimerization of CLIMP-63, that is dependent on palmitoylation. We also show that CLIMP-63 contributes to gentamicininduced apoptosis in proximal tubule cells, likely through dimerization of CLIMP-63. Finally, we identified 14-3-3 proteins as CLIMP-63-binding proteins, and that gentamicin enhances 14-3-3 $\beta$ binding to CLIMP-63 through 14-3-3 $\theta$, having a major role in gentamicin-induced cytotoxicity.

\footnotetext{
${ }^{1}$ Oregon Hearing Research Center, Oregon Health \& Science University, 3181 SW Sam Jackson Park Road, Portland, OR, 97239, USA and ${ }^{2}$ Proteomics Shared Resource, Oregon Health \& Science University, 3181 SW Sam Jackson Park Road, Portland, OR, 97239, USA

${ }^{*}$ Corresponding author: T Karasawa, Oregon Hearing Research Center, Oregon Health \& Science University, 3181 SW Sam Jackson Park Road, Portland, OR 97239, USA. Tel: 503494 2373; Fax: 503494 5656; E-mail: karasawa@ohsu.edu

Keywords: aminoglycosides; gentamicin; CLIMP-63; CKAP4; cytotoxicity; 14-3-3

Abbreviations: GBPs, gentamicin-binding proteins; CLIMP-63, cytoskeleton-linking membrane protein of $63 \mathrm{kDa}$; ER, endoplasmic reticulum; DTT, dithiothreitol; GTTR, gentamicin-Texas Red; MTT, 3-(4,5-dimethylthiazol-2-yl)-2, 5-diphenyltetrazolium bromide; TUNEL, terminal deoxynucleotidyl transferase-mediated dUTP nick end labeling

Received 15.6.10; revised 27.9.10; accepted 14.10.10; Edited by R Mantovani
} 


\section{Results}

To identify GBPs, we used mouse kidney cell lines as described previously. ${ }^{12}$ On the basis of morphological differences, TRPV4 expression as a distal tubule marker, and susceptibility to gentamicin treatment, cell lines of proximal and distal tubule origins were identified. ${ }^{12,20}$ The susceptibility of a proximal tubule cell line, KPT11, to gentamicin-induced toxicity was compared with KDT3, a distal tubule cell line. After 3 days of gentamicin treatment, KPT11 showed significantly lower cell viability compared with KDT3 (Figure 1a). Caspase-3 activity was significantly higher in KPT11 compared with KDT3, after 2 days of gentamicin treatment (Figure 1b). These data confirmed that the proximal tubule cell line KPT11 is more susceptible to gentamicin treatment compared with the distal tubule cell line KDT3, and that cytotoxicity involves apoptotic mechanisms.

Identification of GBPs. GBPs were pulled down from total cell lysates of KPT11 and KDT3 by using gentamicinagarose conjugates, and resolved by SDS gel electrophoresis. Coomassie blue staining revealed that most of the GBP bands were similar in density between these two cell lines (Figure 1c). However, several GBP bands reproducibly had greater density in KPT11 cells compared with KDT3 cells (at least three times out of several electrophoresis runs; Figure 1c). These KPT11-specific GBP bands were excised and analyzed by mass spectrometry. Proteins identified with the highest numbers of unique spectra for each band by mass spectrometric analysis were considered valid GBPs (Supplementary Table S1). These were proteins generally associated with the cytoskeleton (myosin IIA, caldesmon, actin, $\alpha$-tropomyosin), including CLIMP-63 that anchors the ER to the cytoskeleton. Western blotting data confirmed that each of these proteins was pulled down at higher levels in KPT11 than KDT3 cells except for $\alpha$-tropomyosin (Figure 1d). It is possible that tropomyosin bands in the western blot showed multiple tropomyosin isoforms and did not reflect the levels of $\alpha$-tropomyosin. Because of the sequence homology, mass spectrometric analysis could not specifically distinguish between $\beta$ - and $\gamma$-actin isoforms.

CLIMP-63 co-localizes with GTTR in the cell. KPT11 cells were treated with $5 \mu \mathrm{g} / \mathrm{ml}$ GTTR in growth medium for $1 \mathrm{~h}$. Lysosomal fluorescence was quenched to detect GTTR in organelles with low levels of uptake. ${ }^{8,9}$ CLIMP-63 immunofluorescence revealed a high degree of overlapping
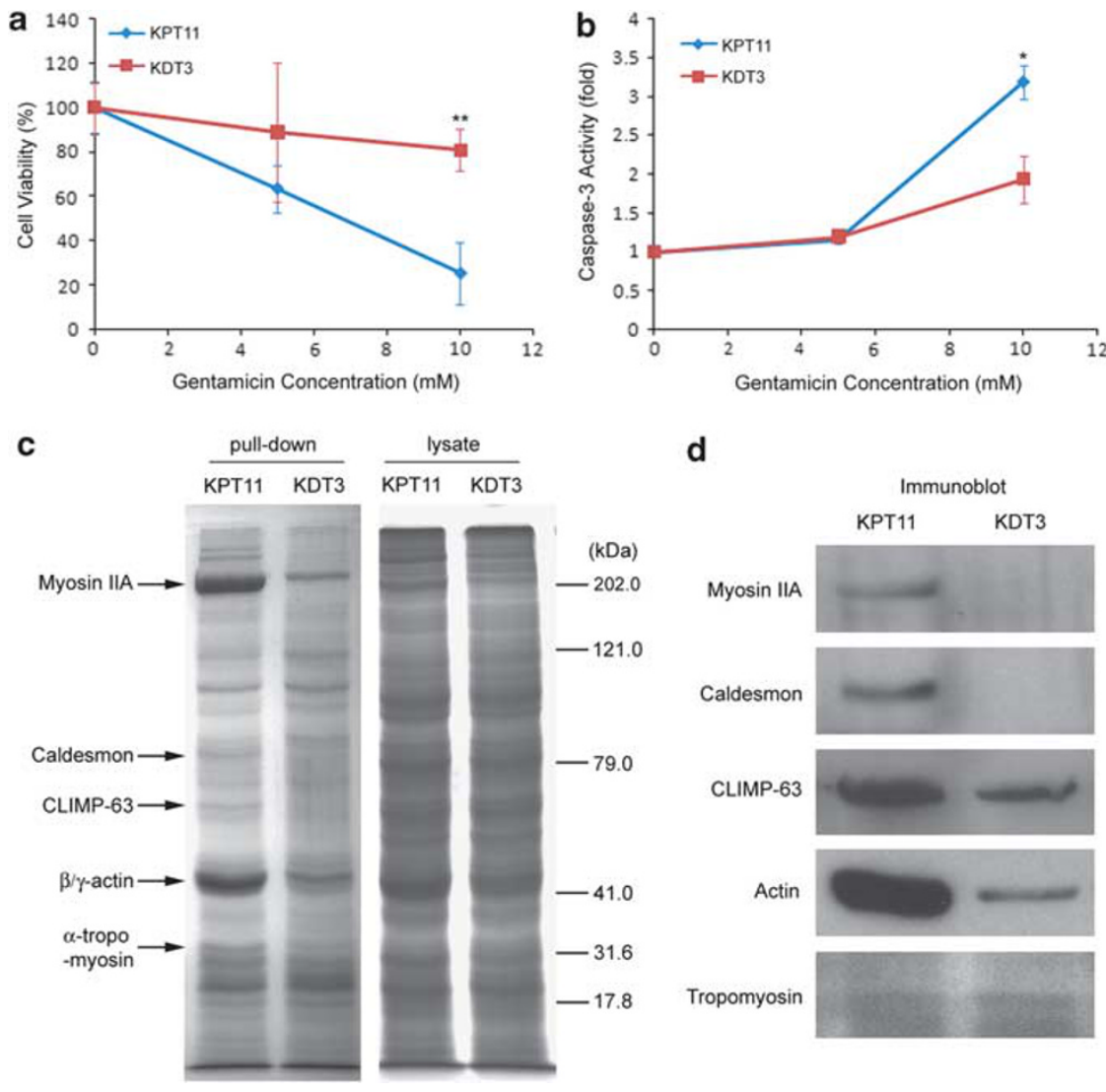

Figure 1 Gentamicin-binding protein identification. (a) Proximal tubule KPT11 cells and distal tubule KDT3 cells were compared for cell viability after gentamicin treatment for 3 days by MTT assay. KPT11 cells had significantly lower viability compared with KDT3 cells following $10 \mathrm{mM}$ gentamicin treatment (** $P<0.01)$. (b) Gentamicin (10 mM) induced significantly more caspase-3 activity in KPT11 cells compared with KDT3 cells $\left({ }^{*} P<0.05\right)$. Data in $\mathbf{a}$ and $\mathbf{b}$ are shown as mean \pm S.D. of three independent experiments. (c) GBPs were pulled down from total cell lysates of KPT11 and KDT3 cells. SDS-polyacrylamide gel electrophoresis followed by Coomassie blue staining revealed several GBP bands specific to KPT11 cells (representative gel from three experiments). Mass spectrometric analysis of peptides from excised gel bands identified these GBPs with the highest numbers of unique peptides. (d) Western blotting on the pull-down samples confirmed the GBPs identified by mass spectrometry 

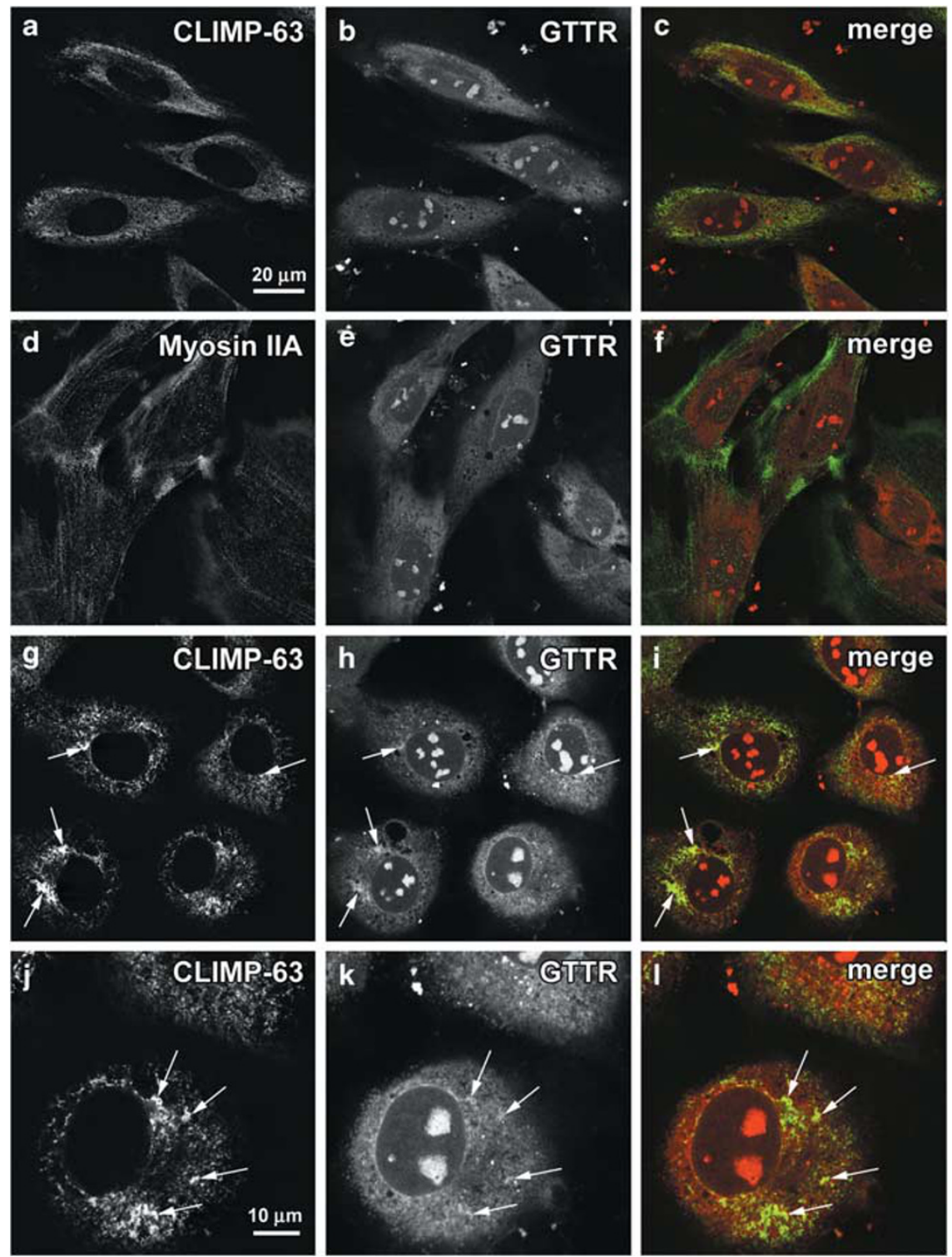

Figure 2 CLIMP-63 co-localizes with GTTR. Cells were treated with GTTR for $1 \mathrm{~h}$ before immunolabeling for GBPs. CLIMP-63 and GTTR had highly overlapping localization (a-c), whereas myosin IIA and GTTR showed no significant co-localization (d-f) in KPT11 cells. GTTR in COS-7 cells was not evenly distributed in the cytoplasm and was highly co-localized with CLIMP-63, as indicated by arrows ( $\mathbf{g}-\mathbf{l})$. Scale bar in $\mathbf{a}$ also applies for $\mathbf{b}-\mathbf{i}(20 \mu \mathrm{m})$. Images $\mathbf{j}-\mathbf{I}$ were magnified from lower- right corner of $\mathbf{g}-\mathbf{i}$, and scale bar in $\mathbf{j}$ also applies for $\mathbf{k}$ and $\mathbf{I}$

localization with GTTR in the cytoplasm (Figures 2a-c). Myosin IIA and caldesmon had distinctively different immunolocalization patterns from CLIMP-63 and little co-localization with GTTR (Figures 2d-f, data not shown). As previous studies on CLIMP-63 were primarily conducted on monkey kidney COS-7 cells, ${ }^{18,19}$ we also examined GTTR and CLIMP-63 localization in COS-7 cells, and observed significant co-localization of GTTR with CLIMP-63 (Figures 2g-l). Although gentamicin may bind to myosin IIA and caldesmon, their weak colocalization with GTTR suggests that their interactions may not have significant roles in inducing toxicity. Therefore, we sought to determine whether CLIMP-63 has a role in gentamicin-induced toxicity in the cell.
Different oligomerization states of CLIMP-63 in kidney tubule cells. Although suggested before, ${ }^{21}$ ubiquitous expression of CLIMP-63 has not been confirmed. CLIMP-63 mRNA expression was detected in all mouse tissues tested by RT-PCR, and was lower in the kidney compared with most other tissues (Figure 3a). CLIMP-63 expression was higher in proximal tubule cell lines (KPT11 and KPT2 ${ }^{12}$ ) compared with distal tubule cell lines (KDT3 and MDCT; ${ }^{22}$ Figure 3b). This was confirmed by western blotting (Figure 3c). TRPV4 expression is a marker for distal tubule cells $^{20}$ (Figure 3c). There was no significant difference between proximal and distal tubule cells in expression levels of calreticulin (CRT), a previously identified GBP. ${ }^{23}$ Remarkably, proximal tubule cells also exhibited a second 
a

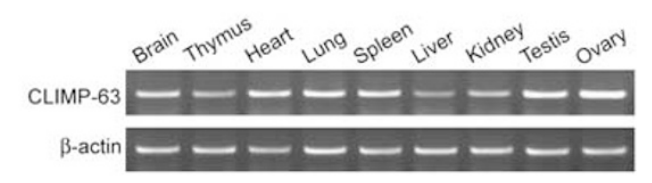

C
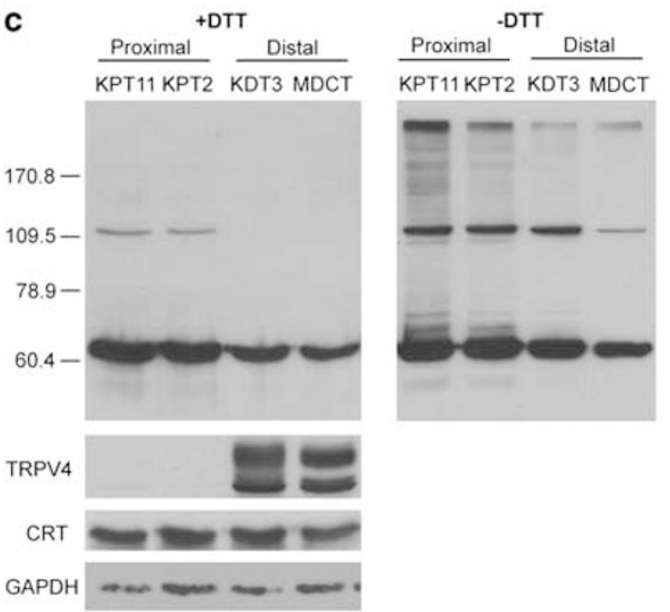

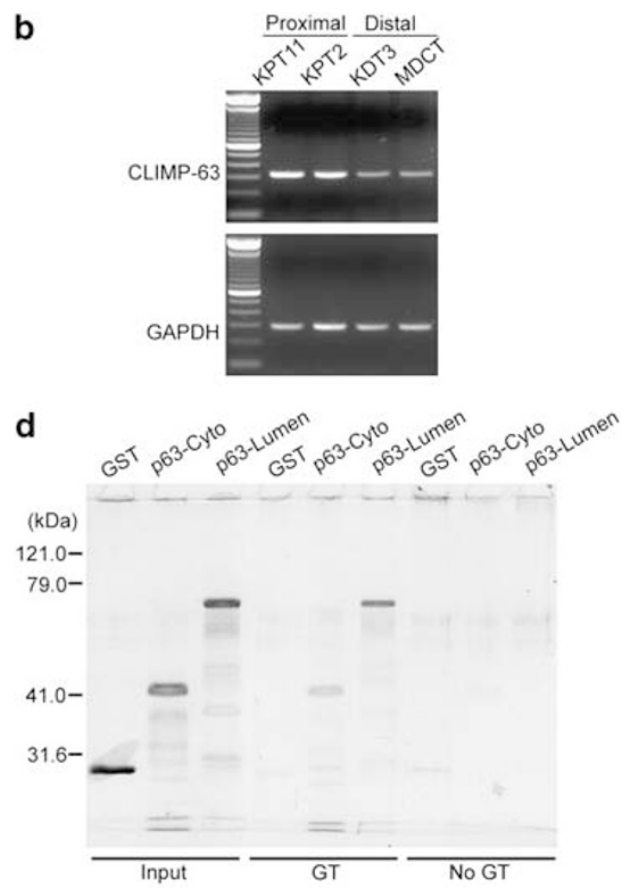

Figure 3 Different expression levels of CLIMP-63, oligomerization of CLIMP-63, and gentamicin binding to the CLIMP-63 lumenal domain. (a) RT-PCR of specified mouse tissues revealed varying CLIMP-63 expression levels. (b) RT-PCR of mouse kidney cell lines show higher levels of CLIMP-63 mRNA expression in proximal tubule cells compared with distal tubule cells. (c, left panel) Proximal, but not distal, tubule cells express DTT-resistant dimers of CLIMP-63, revealed by western blotting. (c, right panel) Without DTT, all samples displayed bands that correspond to dimers and tetramers. (d) The GST fusions of the cytosolic domain (p63-Cyto) and lumenal domain (p63-lumen) had approximate molecular masses of 42 and $75 \mathrm{kDa}$, respectively, as revealed by Coomassie blue staining (shown as 'input'). p63-lumen was efficiently pulled down, whereas negligible GST or weak p63-Cyto proteins were pulled down by gentamicin-agarose (GT). There were no GST fusions pulled down by agarose without gentamicin conjugation (no GT), although a weak GST band was detected

band that corresponds to the dimeric form of CLIMP-63 (Figure 3c). CLIMP-63 forms oligomers that can be detected in non-reducing conditions without dithiothreitol (DTT). ${ }^{24}$ In non-reducing conditions (-DTT), bands were observed $\sim 126$ and $252 \mathrm{kDa}$ in all samples tested (Figure 3c), likely dimers and tetramers of CLIMP-63. The high molecular mass bands in reducing conditions (+DTT) were at the same position as in non-reducing conditions, suggesting that these were also dimers. Resistance of dimers to reducing conditions suggests that these are likely formed by non-disulfide bonding. As mouse fibroblast 3T3 cells also showed similar levels of CLIMP-63 monomer expression as KPT11 cells, but with negligible levels of dimers in western blots, these DTT-resistant dimers are not likely due to high levels of CLIMP-63 expression (Supplementary Figure $\mathrm{S} 1 \mathrm{~A}$ and $\mathrm{B}$ ).

The lumenal domain of CLIMP-63 forms a coiled-coil structure that is required for oligomerization. ${ }^{19}$ As only proximal tubule cells showed the DTT-resistant dimers in western blots, CLIMP-63 oligomerization states in kidney proximal and distal tubule cells are likely different. To determine whether CLIMP-63 interacts with gentamicin at the $\mathrm{N}$-terminal cytosolic domain or the $\mathrm{C}$-terminal lumenal domain, GST fusion proteins of CLIMP-63 domains were generated for gentamicin-agarose pull-down assays. Gentamicin-agarose pulled down the lumenal domain of CLIMP-63 in significantly high amounts, as revealed by Coomassie blue staining (Figure $3 d$ ). The cytosolic domain was only weakly pulled down by gentamicin-agarose, but this was likely non-specific binding, as GST alone was also pulled down at a similar level by agarose without gentamicin. These data suggest that CLIMP-63 and gentamicin interact with each other within the ER lumen.

A cochlear cell line expresses CLIMP-63 with a modification similar to proximal tubule cells. In the kidney, proximal tubule cells express greater levels of monomeric CLIMP-63 and DTT-resistant dimers than distal tubule cells. The inner ear is another primary site for aminoglycoside toxicity. We analyzed cochlear cell lines for the presence of CLIMP-63 dimers. HEI-OC1 (organ of Corti cell line) and SV-k1 (stria vascularis cell line) are cochlear cell lines of similar genetic background from transgenic mice, ${ }^{25,26}$ with cells transformed by a temperature-sensitive mutant of large T-antigen. These cells grow rapidly at $33^{\circ} \mathrm{C}$ (permissive), but adopt a more differentiated state at $39^{\circ} \mathrm{C}$ (non-permissive). Interestingly, a high level of the non-reducible CLIMP-63 dimers appeared in HEI-OC1 cells at the non-permissive condition $\left(39^{\circ} \mathrm{C}\right)$ but not at the permissive condition $\left(33^{\circ} \mathrm{C}\right.$; Figure $\left.4 \mathrm{a}\right)$. RT-PCR showed that there was no significant difference in expression of CLIMP-63 at the transcription level in $\mathrm{HEI}-\mathrm{OC} 1$ cells grown at non-permissive conditions, although $\beta$-actin expression was reduced (Figure $4 \mathrm{~b}$ ). As the non-permissive condition did not enhance dimer formation in SV-k1 cells, dimerization is likely organ of Corti specific, and could also occur in vivo. 
a

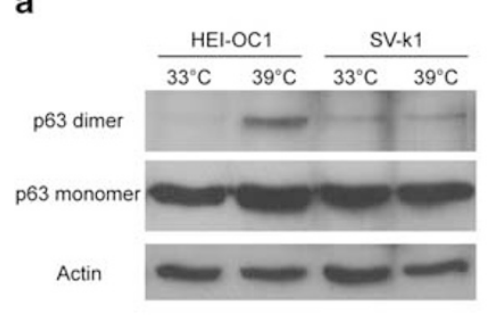

b

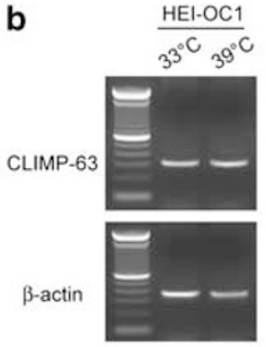

C
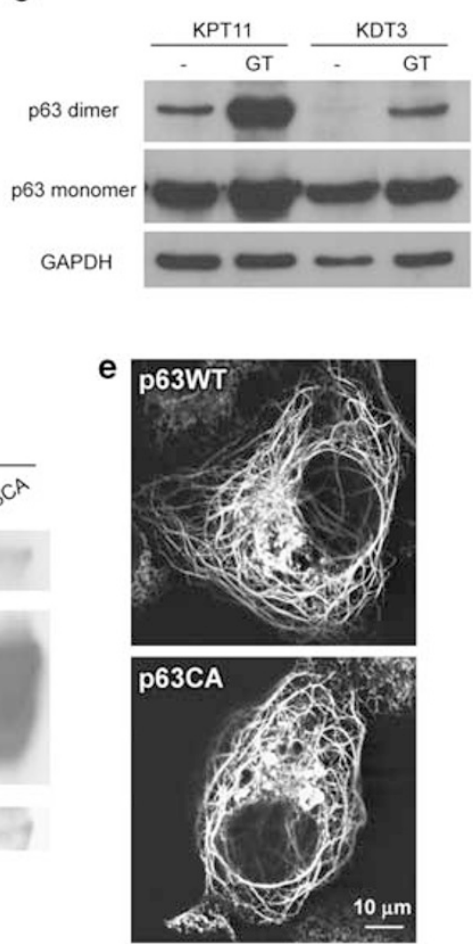

Figure 4 CLIMP-63 in a cochlear organ of Corti cell line forms the DTT-resistant dimers, and gentamicin induces the dimerization of the CLIMP-63 that is dependent on palmitoylation. (a) In the non-permissive condition $\left(39^{\circ} \mathrm{C}\right)$, the organ of Corti HEI-OC1 cell line expressed the DTT-resistant dimer form of CLIMP-63 in western blots. However, there was minimal dimer induction in stria vascularis SV-k1 cells in either condition. (b) CLIMP-63 gene expression levels were not altered in HEI-OC1 cells in the non-permissive condition, confirmed by RT-PCR. (c) After gentamicin treatment at $5 \mathrm{mM}$ for 3 days, both KPT11 and KDT3 expressed higher levels of the DTT-resistant dimers, and higher monomer levels in KPT11 cells in western blots. (d) Cells (293T) transfected with the wild-type CLIMP-63 (p63WT) formed the DTT-resistant dimers, but those with palmitoylation-incompetent mutants C100S and C100A (p63CS and p63CA) showed reduced amounts of the dimers compared with the wild type. Gentamicin treatment increased the dimer level only in cells transfected with the wild-type CLIMP-63. (e) COS-7 transfected with the CLIMP-63 wild type (p63WT) or C100A mutant (p63CA) showed microtubule associated expression. The C100S mutant also showed similar expression pattern (data not shown)

Gentamicin induces DTT-resistant dimerization of CLIMP-63. To determine whether gentamicin affects CLIMP-63, we treated KPT11 and KDT3 cells with gentamicin. After 3 days of gentamicin treatment, KPT11 cells showed increased levels of dimers, and possibly monomers of CLIMP-63, by western blotting (Figure 4c). Importantly, KDT3 cells that normally do not exhibit the non-reducible dimers also contained a dimer band after gentamicin treatment (Figure 4c), suggesting that gentamicin induces DTT-resistant CLIMP-63 dimers. This also implicates that CLIMP-63 dimerization is associated with gentamicininduced cytotoxicity.

CLIMP-63 palmitoylation is required for the gentamicininduced DTT-resistant dimerization. A post-translational modification, palmitoylation, has been associated with CLIMP-63 subcellular localization. ${ }^{27,28}$ To test whether palmitoylation and DTT-resistant dimerization of CLIMP-63 are related, we transfected wild-type and palmitoylationincompetent mutants of CLIMP-63 (C100S and C100A) into human embryonic kidney $293 \mathrm{~T}$ cells, and analyzed the state of CLIMP-63 by western blotting. Overexpression of wild-type CLIMP-63 resulted in a band corresponding to the dimer species, but the mutants did not show significant levels of dimers (Figure 4d). Interestingly, CLIMP-63-transfected
293T cells treated with gentamicin for 3 days increased the amount of the DTT-resistant dimers in the wild-type transfected cells, but not mutant transfected cells. There was no significant difference in subcellular localization between the transfected wild-type CLIMP-63 or either mutant in COS-7 cells (Figure 4e). Although the monoclonal antibody also detected endogenous CLIMP-63 (Figure 2j), the localization patterns of endogenous and transfected CLIMP-63 were distinctively different (Figure 2j and Figure $4 \mathrm{e}) .{ }^{18}$ Therefore, gentamicin-induced dimerization of CLIMP-63 requires palmitoylation, and the inability of mutant to form the dimer is not because of the changes in subcellular localization.

CLIMP-63 contributes to gentamicin-induced toxicity in kidney proximal tubule cells. To determine whether CLIMP-63 in proximal tubule cells contributes to gentamicin cytotoxicity, two sets of siRNA for CLIMP-63 and scrambled control were designed and generated. Transfection of CLIMP-63 siRNA reduced protein expression levels of the DTT-resistant dimer in KPT11 cells, although there was no apparent reduction in the monomer form (Figure 5a). RT-PCR showed that siRNA effectively reduced CLIMP-63 transcripts (Figure 5b). CLIMP-63 siRNA transfection into KPT11 resulted in $\sim 5-10 \%$ reduction in cell number, after 3 days of transfection, compared with cells transfected with 
a
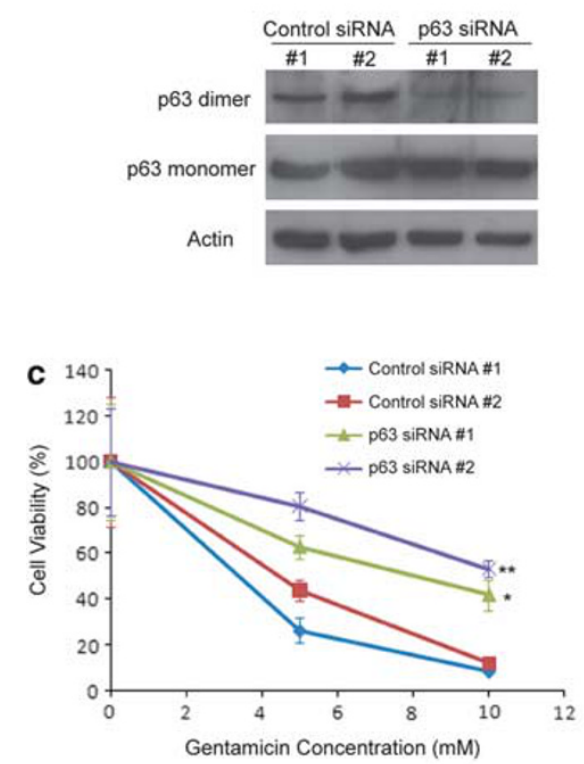
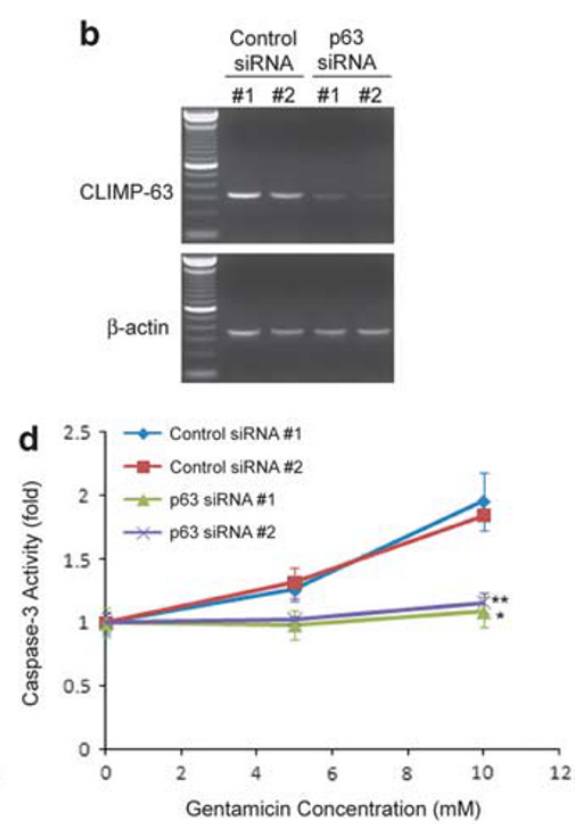

e
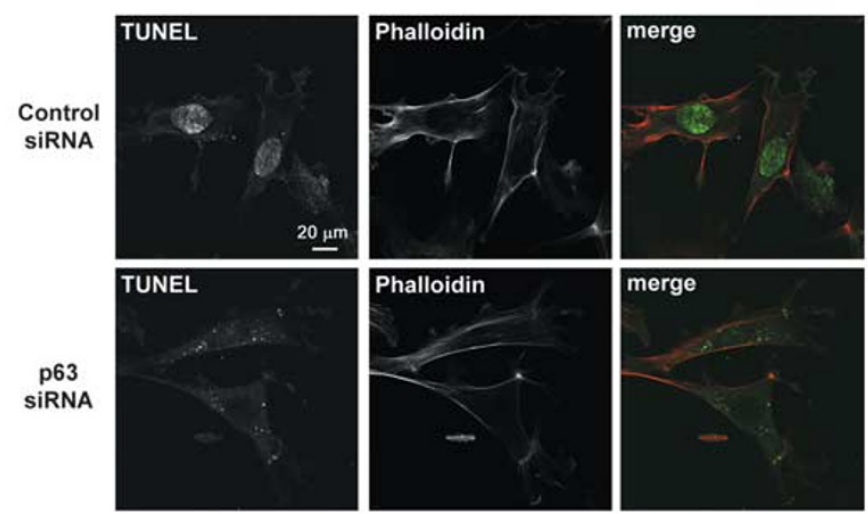

Figure 5 Reducing CLIMP-63 dimer expression by siRNA transfection enhances cellular resistance to gentamicin-induced toxicity. (a) Two sets of siRNA for CLIMP-63 and scrambled control were transfected into KPT11 cells. Although the monomer form of CLIMP-63 (p63 monomer) did not show any apparent reduction, the DTT-resistant dimer form (p63 dimer) was reduced by siRNA for CLIMP-63 (p63 siRNA no. 1 and no. 2) as shown by western blotting. (b) CLIMP-63 gene knockdown by siRNA was confirmed by RT-PCR. (c) After 3 days of gentamicin treatment, cell viability was higher in CLIMP-63 siRNA-transfected cells compared with control, as determined by MTT assay $\left({ }^{*} P<0.05\right.$ between control and p63 siRNA no. $1,{ }^{* *} P<0.01$ between control and p63 siRNA no. 2). (d) Caspase-3 activity was suppressed in cells transfected by CLIMP-63 siRNA after $24 \mathrm{~h}$ of gentamicin treatment $\left({ }^{*} P<0.05\right.$ between control and p63 siRNA no. $1,{ }^{\star *} P<0.01$ between control and p63 siRNA no. 2). Data in (c and d) are shown as mean \pm S.D. of three independent experiments. (e) DNA strand breaks were detected by TUNEL reaction in control siRNA-transfected KPT11 after $24 \mathrm{~h}$ of gentamicin treatment, but not in CLIMP-63 siRNA-transfected cells. Phalloidin labeled the filamentous actin cytoskeleton, allowing visualization of cell morphology

control siRNA (data not shown). However, after 3 days of gentamicin treatment, CLIMP-63 siRNA-transfected KPT11 cells had higher cell viability compared with control cells (Figure 5c). To measure caspase-3 activity, proteins were extracted from siRNA-transfected KPT11 cells after $24 \mathrm{~h}$ of gentamicin treatment. Strikingly, CLIMP-63 siRNA transfection suppressed gentamicin-dependent caspase-3 activity completely, whereas $10 \mathrm{mM}$ gentamicin increased caspase-3 activity twofold in control siRNA-transfected cells (Figure $5 d$ ). Gentamicin-induced caspase-3 activity continued to increase after 12 and $24 \mathrm{~h}$ (Supplementary Figure S1C). Confirmation that caspase- 3 activation was due to apoptosis was provided by terminal deoxynucleotidyl transferase-mediated dUTP nick end labeling (TUNEL) assays. After $24 \mathrm{~h}$ of gentamicin treatment, $\sim 10 \%$ of control siRNA-transfected KPT11 cells were positive for TUNEL labeling, whereas most CLIMP-63 siRNA-transfected cells showed no labeling in the nuclei $(<1 \%$ of the cell population; Figure $5 e)$.

Experiments with KDT3 cells were also performed using CLIMP-63 siRNA transfection and gentamicin treatment, to compare the differences between kidney proximal and distal tubule cells. The efficacy of siRNA transfection was confirmed by RT-PCR (Supplementary Figure S1D). CLIMP-63 siRNAtransfected cells did not show significantly different cell viability or caspase-3 activity compared with control siRNAtransfected cells after gentamicin treatment, although there appeared to be minor effects in reducing the toxicity (Supplementary Figure S1E, F). These data also suggest that CLIMP-63-dependent gentamicin cytotoxicity requires the DTT-resistant dimer of the protein. 


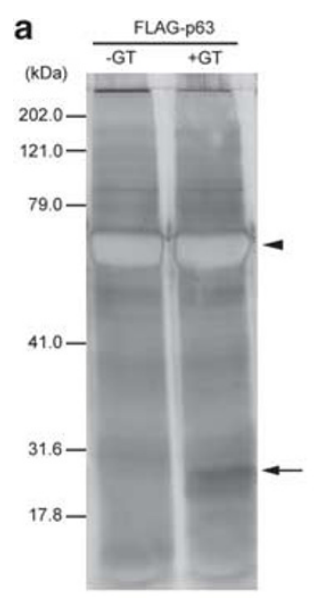

b

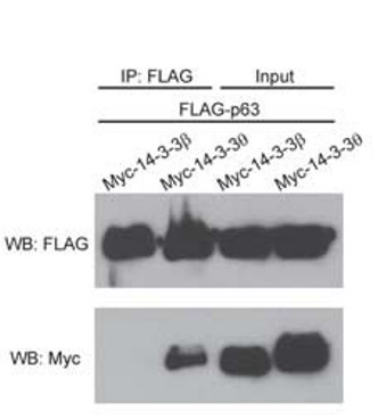

e

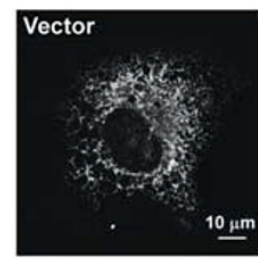

d
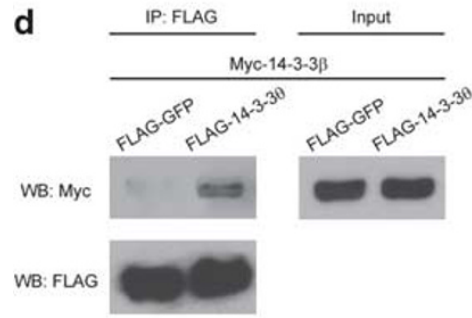
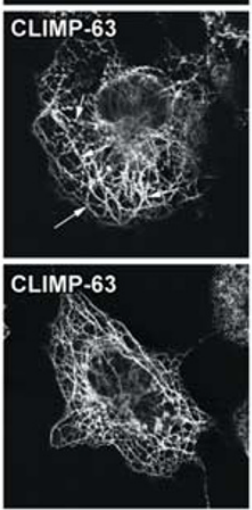
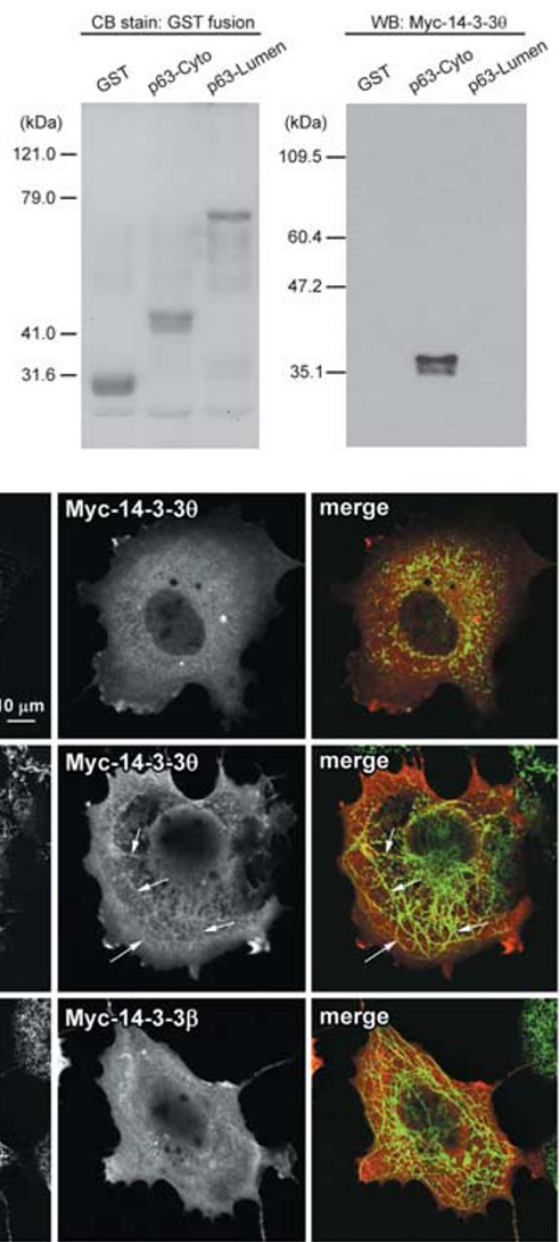

Figure 6 CLIMP-63 binds to 14-3-30, which in turn binds to 14-3-3 $\beta$. (a) Immunoprecipitation was conducted on cell lysate from FLAG-tagged CLIMP-63 (FLAG-p63)transfected 293T cells, using anti-FLAG antibody. SDS-gel electrophoresis followed by silver staining revealed a band specific to cells treated with $5 \mathrm{mM}$ gentamicin for $24 \mathrm{~h}$ ( + GT), which was not present in no gentamicin control (-GT), as indicated by an arrow. Mass spectrometry on the band identified 14-3-3 $\beta$ and $\theta$. FLAG-p63 band is indicated by an arrowhead. The band was white because of the silver staining saturation effect. (b) 293T cells were co-transfected with FLAG-p63 and Myc-tagged 14-3-3 $\beta$ or $\theta$ (Myc-14-3-3 $\beta$ or $\theta$ ), and immunoprecipitation using anti-FLAG antibody was performed after $24 \mathrm{~h}$ of incubation. FLAG-p63 was co-immunoprecipitated with Myc-14-3-30, but not $\beta$. (c) GST fusion pull-down assay was performed on cell lysate from Myc-14-3-30-transfected 293T cells. Coomassie blue staining of GST fusions followed by SDS-gel electrophoresis confirmed that comparable levels of GST fusions were used (left panel). Western blotting on the pull-down samples showed that Myc-14-3-3 $\theta$ binds only to the cytosolic domain of CLIMP-63 (p63-Cyto), and not to GST or the lumenal domain (p63-lumen). (d) FLAG-tagged GFP or 14-3-(FLAG-GFP or 14-3-30) was co-transfected with Myc-14-3-3 $\beta$. Immunoprecipitation using anti-FLAG antibody also pulled down Myc-14-3-3 $\beta$, suggesting that 14-3-3 $\theta$ and $\beta$ binds with each other, probably forming a dimer. (e) COS-7 cells were co-transfected with CLIMP-63 and Myc-14-3-30 or $\beta$. In the absence of exogenous CLIMP-63 expression, Myc-14-3-3 $\theta$ was localized in the cytoplasm, and co-localization with endogenous CLIMP-63 was not very clear (upper panels). CLIMP-63 overexpression altered localizations of not only CLIMP-63 but also Myc-14-3-30, more distributed along peripheral sites of cells (middle panels). There was also significant co-localization between CLIMP-63 and Myc-14-3-30, as indicated by arrows. Myc-14-3-3 $\beta$ did not show clear co-localization with overexpressed CLIMP-63, but still altered localization, possibly through endogenous 14-3-30 protein (lower panels)

CLIMP-63 binds to 14-3-3 proteins and gentamicin enhances their interaction. CLIMP-63 may induce gentamicin cytotoxicity through binding to other proteins. To identify such proteins, FLAG-tagged CLIMP-63 (FLAGp63) was transfected into 293T cells, and cells were treated with gentamicin for $24 \mathrm{~h}$. FLAG-p63 was immunoprecipitated with anti-FLAG antibody, and the samples were analyzed by SDS-gel electrophoresis followed by silver staining. A band specific to cells treated with gentamicin was detected in the $28 \mathrm{kDa}$ region (Figure $6 \mathrm{a}$ ). Mass spectrometric analysis of this band identified a number of proteins, and those that fit the molecular mass of $\sim 28 \mathrm{kDa}$ were 14-3-3 proteins. On the basis of results from two separate experiments, these were identified as $14-3-3 \theta, 14-3-3 \beta, 14-3-3 \zeta$, and 14-3-3 $\varepsilon$. As 14-3-3 $\theta$ and $\beta$ were the predominant $14-3-3$ proteins to be identified, we explored their interactions with CLIMP-63 and their possible roles in gentamicin-induced apoptosis.

To confirm the interactions between CLIMP-63 and 14-3-3 proteins, 293T cells were co-transfected with FLAG-p63 and Myc-tagged 14-3-3 $\beta$ and $\theta$ (Myc-14-3-3 $\beta$ and $\theta$ ). FLAG immunoprecipitation was performed on cell lysate from transfected cells, and samples were analyzed by western blotting. Unexpectedly, only Myc-14-3-3 $\theta$ was co-immunoprecipitated with FLAG-p63 (Figure 6b). CLIMP-63 interaction with 14-3-3 $\theta$ was further confirmed by GST fusion pull-down assay using GST fusion proteins of CLIMP-63 domains. GST 
a
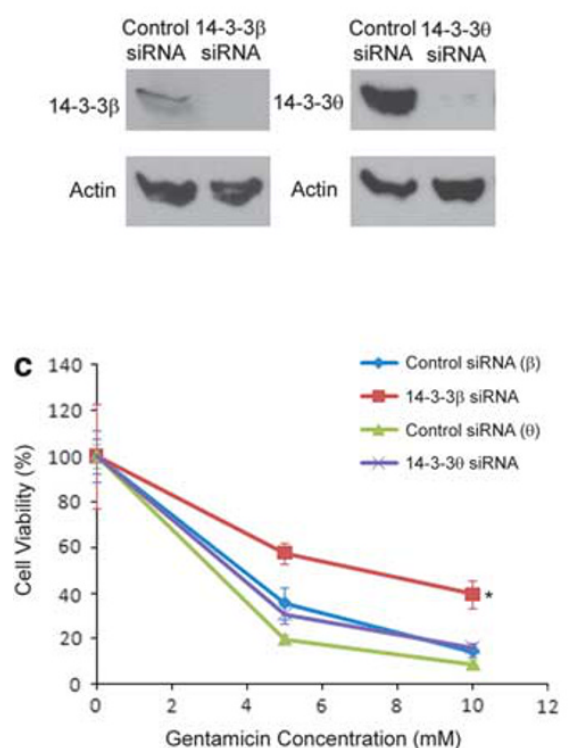
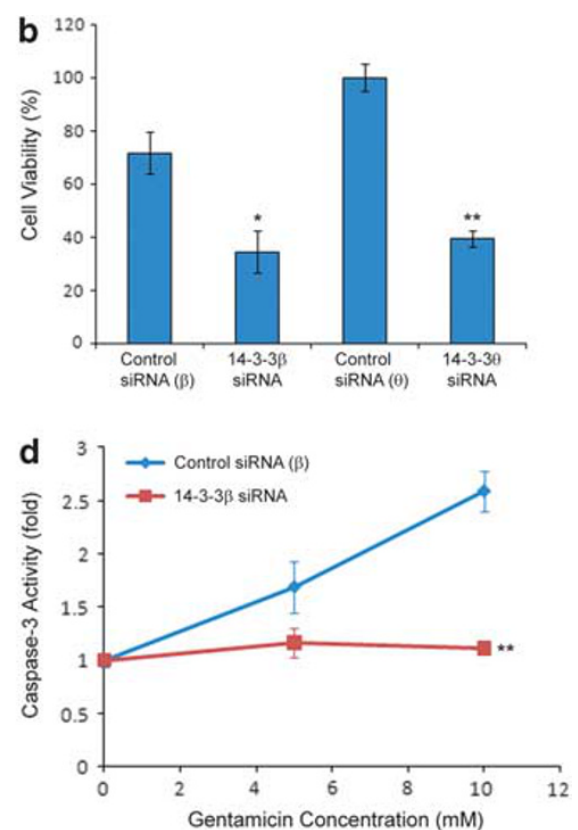

Figure 7 14-3-3 $\beta$ protein is required for CLIMP-63-dependent gentamicin-induced cytotoxicity. (a) KPT11 cells were transfected with siRNA for 14-3-3 $\beta$ and $\theta$, and cells were lysed after 3 days. Western blotting showed that siRNA could knockdown protein expression at high efficiencies. (b) In MTT assay, 14-3-3 siRNA-transfected KPT11 cells exhibited lower cell viability compared with control siRNA-transfected cells $\left({ }^{\star} P<0.05,{ }^{* \star} P<0.01\right.$ against respective control). (c) Gentamicin treatment decreased viability of cells transfected with all siRNA after 3 days, but those transfected with 14-3-3 $\beta$ siRNA were resistant to gentamicin $\left({ }^{*} P<0.05\right)$. Although 14-3-3 $\beta$ siRNA-transfected cells showed lower viability compared with control, the MTT assay raw data at $10 \mathrm{mM}$ gentamicin showed that more cells were viable than control cells $\left(A_{540}=0.47 \pm 0.07\right.$ versus $0.35 \pm 0.06$ ). (d) Caspase-3 activity did not increase in cells transfected with 14-3-3 $\beta$ siRNA after $24 \mathrm{~h}$ of gentamicin treatment, as opposed to cells with control siRNA, which showed $>2.5$-fold increase at $10 \mathrm{mM}$. Without gentamicin treatment, there was no significant difference in caspase-3 activity between 14-3-3 $\beta$ siRNA and control cells (14-3-3 $\beta$ siRNA: $A_{405}=0.09 \pm 0.05$, control: $\left.A_{405}=0.12 \pm 0.07\right)$. Data in (b, $\mathbf{c}$, and $\left.\mathbf{d}\right)$ are shown as mean \pm S.D. of three independent experiments

fusion protein-bound glutathione was mixed with cell lysate from 293T cells transfected with Myc-14-3-30, and binding proteins pulled down. SDS-gel electrophoresis followed by western blotting showed that the cytosolic domain of CLIMP-63 binds to Myc-14-3-30 (Figure 6c).

As 14-3-3 $\beta$ was identified as CLIMP-63-binding protein in mass spectrometry but not in co-immunoprecipitation experiments, we explored the possibility that $14-3-3 \beta$ interacts with CLIMP-63 through 14-3-3 $\theta$ by forming a heterodimer between 14-3-3 $\beta$ and $\theta$. Myc-14-3-3 $\beta$ was co-transfected with FLAGtagged GFP or 14-3-3 $\theta$ (FLAG-GFP or FLAG-14-3-3 $\theta$ ) into 293T cells, and immunoprecipitation with anti-FLAG antibody and western blotting showed that FLAG-14-3-30, but not FLAG-GFP, co-immunoprecipitated Myc-14-3-3 $\beta$ (Figure 6d). A reciprocal experiment using FLAG-14-3-3 $\beta$ and Myc-14-3-3 $\theta$ also confirmed that $14-3-3 \beta$ interacts with $14-3-3 \theta$ (data not shown).

To determine whether CLIMP-63 interacts with 14-3-3 proteins in the cell, CLIMP-63 was co-transfected with Myc14-3-3 $\theta$ or $\beta$ into COS-7 cells, and immunofluorescence was performed after $24 \mathrm{~h}$. The ER localization of endogenous CLIMP-63 was overlapped with Myc-14-3-3 $\theta$ and $\beta$, but their co-localization was not very clear (Figure 6e, upper panels, and data not shown). CLIMP-63 transfection re-organized the localization of the protein as shown before (Figure 4e), and most importantly, Myc-14-3-30 localization was altered and there was significant co-localization with CLIMP-63 (Figure $6 e$, middle panels). Myc-14-3-3 $\beta$ localization was also changed by CLIMP-63 transfection, although there was no clear indication that they were co-localized (Figure 6e, lower panels). It is possible that exogenous CLIMP-63 changed Myc-14-3-3 $\beta$ localization through endogenous 14-3-3 $\theta$ or other $14-3-3$ proteins that bind to CLIMP-63.

Protein 14-3-3 $\beta$ contributes to gentamicin-induced cytotoxicity. Having established that 14-3-3 proteins are CLIMP-63-binding proteins, we investigated the possibility that these proteins have a role in gentamicin-induced apoptotic mechanisms. We designed and obtained siRNA duplexes for $14-3-3 \beta$ and $\theta$, and transfection into KPT11 cells confirmed that these siRNA efficiently knocked down protein expression (Figure 7a). Without gentamicin treatment, siRNA for each 14-3-3 lowered cell viability, approximately half of those transfected with control siRNA (Figure $7 b$ ). This is consistent with previous studies that 14-3-3 proteins are crucial for cellular growth. ${ }^{29}$ Gentamicin treatment for 3 days further reduced cell viability of 14-3-3 siRNA-transfected cells, as well as control cells. However, normalization of cell viability with respect to gentamicin treatment revealed that $14-3-3 \beta$ siRNA-transfected cells showed significantly more viability compared with other siRNA-transfected cells (Figure 7c). This was not because of the low basal viability by 14-3-3 siRNA, because the raw cell viability assay data at $10 \mathrm{mM}$ gentamicin showed that significantly more 14-3-3 $\beta$ siRNA-transfected cells were viable than control cells $\left(A_{540}=0.47 \pm 0.07\right.$ versus $\left.0.35 \pm 0.06 ; \quad P<0.05\right)$. Strikingly, there was no increase in caspase-3 activity in 14-3-3 $\beta$ siRNA-transfected cells after $24 \mathrm{~h}$ of gentamicin treatment (Figure 7d). Therefore, we concluded that 14-3-3 $\beta$ has a role in gentamicin-induced apoptosis. 


\section{Discussion}

Although several mechanisms for aminoglycoside-induced toxicity have been described, none has been fully elucidated. Importantly, most studies concerning aminoglycoside toxicity address the mechanisms but overlook tissue specificity. For example, aminoglycosides binding to ribosomal RNA could induce toxicity, but why do the kidney and cochlea have greater susceptibility to the drug? We hypothesized that differences in protein expression may be responsible for the susceptibility of particular cell types in the kidney and cochlea, and that aminoglycosides must interact with intracellular proteins to trigger cell death mechanisms, particularly apoptosis. To identify proteins that bind to aminoglycosides, we used a gentamicin-agarose conjugation technique similar to those reported previously, ${ }^{23,30}$ with an advantage of including a spacer between gentamicin and agarose to reduce steric hindrance of binding sites.

Kidney proximal tubule cells are more susceptible to gentamicin compared with kidney distal tubule cells. Previously identified GBPs, CRT, and HSP73, are ubiquitously expressed in kidney cells, and thus unlikely to be the GBPs responsible for gentamicin susceptibility in proximal tubule cells. ${ }^{23,30,31}$ The GBP pull-down assay revealed greater expression of several GBPs in proximal tubule cells compared with distal tubule cells. Mass spectrometric analysis identified these proteins as mostly cytoskeleton-associated proteins. To identify GBPs that may have physiological relevance, we determined the intracellular localization of GBPs and fluorescent dye-tagged gentamicin (GTTR), previously used to identify cellular mechanisms of gentamicin uptake. ${ }^{12,32}$ As well documented by others, ${ }^{9}$ GTTR was localized mostly in cytoplasm, with some punctate labeling, likely in endosomes and in the ER. Subcellular localization analysis of these GBPs determined that only CLIMP-63 had significant overlapping localization with GTTR.

CLIMP-63 has a leucine zipper motif in its lumenal domain, ${ }^{28}$ the gentamicin-binding site identified in this study, and it is predicted that CLIMP-63 forms a dimer similar to other proteins with leucine zippers. Although examining the western blots of CLIMP-63 derived from proximal and distal tubule-derived cells, we found significant amounts of disulfide-linked dimers. Furthermore, CLIMP-63 from proximal cells was unique as there were also non-disulfide-linked dimers. Similar nondisulfide-linked CLIMP-63 dimers were also found in a cell line derived from the cochlear organ of Corti, a region also susceptible to gentamicin toxicity. Interestingly, gentamicin treatment increased the formation of these non-disulfide-linked dimers. How these non-disulfide-linked dimers have a role in gentamicin-induced cytotoxicity is unclear, but our data suggest that it likely changes CLIMP-63 structure at the cytosolic domain, because a mutation of the palmitoylation site in the cytosolic domain inhibited the dimerization induced by gentamicin.

As a first step to elucidate gentamicin-induced cytotoxicity mechanisms of CLIMP-63, we used FLAG immunoprecipitation and identified 14-3-3 proteins as CLIMP-63 binding proteins. Co-immunoprecipitation and in vitro binding assays showed that $14-3-3 \theta$ but not $14-3-3 \beta$ directly binds to CLIMP-63, and the binding site on CLIMP-63 is the cytosolic domain. We also demonstrated that 14-3-3 $\beta$ and $\theta$ bind with each other, likely forming a heterodimer. ${ }^{33,34}$ As $14-3-3 \beta$ protein was identified as a CLIMP-63-binding protein by mass spectrometry, it is likely that it binds to CLIMP-63 through 14-3-3 $\theta$ or another 14-3-3 family protein. Interactions between CLIMP-63 and 14-3-3 proteins were also confirmed by immunofluorescence.

Finally, to test whether CLIMP-63 expression itself affects drug susceptibility of the cells, we used siRNA transfection instead of protein overexpression, because for most proteins, overexpression itself can be highly toxic to cells. Conveniently, the siRNA much reduced the dimer levels of CLIMP-63 in KPT11 cells, but not monomer levels. Thus, CLIMP-63 siRNA-transfected KPT11 cells could be considered similar to distal tubule cells. Cell viability measurement, caspase-3 assays and TUNEL staining revealed that CLIMP-63 siRNAtransfected KPT11 showed higher resistance to gentamicin treatment compared with control siRNA-transfected cells. The lack of caspase-3 activity observed in CLIMP-63 siRNAtransfected KPT11 cells after treatment with gentamicin is particularly significant because it suggests that gentamicininduced caspase-3 activation requires CLIMP-63 dimerization. However, CLIMP-63 siRNA-transfected KPT11 cells are likely to be susceptible to other cell death mechanisms induced by gentamicin, such as necrosis and caspase3-independent apoptosis. ${ }^{17}$

To determine whether 14-3-3 proteins have a role in gentamicin-induced cytotoxicity, we also designed and obtained siRNA for $14-3-3 \beta$ and $\theta$. Without gentamicin treatment, these siRNA reduced cellular growth by $\sim 50 \%$, as expected because of known 14-3-3 functions in the cell cycle. However, normalization to reveal the effects of gentamicin demonstrated that $14-3-3 \beta$ protein significantly contributes to gentamicin-induced cytotoxicity, particularly with increased caspase- 3 activity that leads to apoptosis. Although it is not clear why knockdown of 14-3-3 $\theta$ did not attenuate gentamicininduced cytotoxicity, it is possible that $14-3-3 \beta$ is the primary 14-3-3 protein that has a mechanistic role in gentamicin toxicity, and it can bind to CLIMP-63 indirectly through a 14-3-3 protein other than 14-3-3 $\theta$, to induce the signaling mechanism. 14-3-3 and $\varepsilon$ proteins are candidates because these were also identified as CLIMP-63-binding proteins.

Although the physiological functions of CLIMP-63 in the cell are not well characterized, especially compared with 14-3-3 proteins, previous studies report that CLIMP-63 functions in cellular proliferation as CLIMP-63 expression is upregulated by a cyclin-dependent kinase inhibitor olomoucine. ${ }^{35} \mathrm{DHHC2}$, a palmitoyl acryltransferase that palmitoylates CLIMP-63, also regulates cellular proliferation through antiproliferative factor and CLIMP-63. ${ }^{28}$ It is possible that gentamicin induces cell death by interfering with cellular proliferation control through CLIMP-63 and 14-3-3 proteins. Future studies on CLIMP-63 and 14-3-3 proteins with gentamicin and other aminoglycosides could provide more insight into drug-induced toxicity mechanisms.

\section{Materials and Methods}

Antibodies. Primary antibodies used in this study are: myosin IIA polyclonal antibody (pAb; Covance, Princeton, NJ, USA), caldesmon monoclonal antibody (mAb; C21, Sigma, St. Louis, MO, USA), CLIMP-63 pAb (a generous gift from Dr. Jack Rohrer, Zurich University of Applied Sciences, Switzerland) and mAb (G1/296, Alexis Biochemicals, San Diego, CA, USA), actin pAb (Sigma, cat. no. A2103), tropomyosin 
pAb (E-17, Santa Cruz Biotechnology, Santa Cruz, CA, USA), TRPV4 pAb (a generous gift from Dr. Stefan Heller, Stanford University, Stanford, CA, USA), CRT pAb (Affinity BioReagents, Golden, CO, USA), GAPDH mAb (GAPDH-71.1, Sigma), FLAG mAb (M2, Sigma), Myc mAb (9E10, Sigma), 14-3-3 $\beta$ mAb (60C10, Santa Cruz Biotechnology), and 14-3-30 mAb (3B9, Santa Cruz Biotechnology).

Cell culture. Mouse kidney primary cultures were generated as described previously. ${ }^{12}$ COS-7, 293T, MDCT, ${ }^{22}$ and NIH-3T3 cells as well as the kidney cell lines we generated (KPT11, KPT2, ${ }^{12} \mathrm{KDT} 3$ ) were maintained at $37^{\circ} \mathrm{C}, 5 \% \mathrm{CO}_{2}$. $\mathrm{HEI}-\mathrm{OC} 1$ and SV-k1 cells (generous gifts from Dr. Federico Kalinec, House Ear Institute, Los Angeles, CA, USA) were maintained at $33^{\circ} \mathrm{C}$ with $10 \% \mathrm{CO}_{2}$ (for the permissive condition), or $39^{\circ} \mathrm{C}$ with $5 \% \mathrm{CO}_{2}$ (for the non-permissive condition). All cells were maintained in DMEM with $10 \%$ FBS.

Gentamicin-agarose pull-down assay. Cells were lysed in buffer containing $150 \mathrm{mM} \mathrm{NaCl}, 50 \mathrm{mM}$ Tris, pH 7.5, $5 \mathrm{mM}$ EDTA, 1\% Triton X-100 with protease inhibitors, and centrifuged $(14000 \mathrm{~g}, 10 \mathrm{~min})$ at $4^{\circ} \mathrm{C}$ to remove cell debris. Protein concentration was adjusted to $1 \mathrm{mg} / \mathrm{ml}$ for all samples. For gentamicinagarose preparation, $100 \mu \mathrm{l}$ Affi-Gel 10 (Bio-Rad, Hercules, CA, USA) was washed with ice-cold water three times and resuspended in $1 \mathrm{ml}$ HEPES ( $\mathrm{pH} 7.0)$, in which $20 \mathrm{mg}$ of gentamicin had been dissolved. The suspension was incubated at $4^{\circ} \mathrm{C}$ overnight. The residual reactive sites on the beads were blocked by $1 \mathrm{M}$ ethanolamine $(\mathrm{pH} 8.0)$ for $1 \mathrm{~h}$, and the beads were washed with PBS three times and stored at $4^{\circ} \mathrm{C}$ until use. The gentamicin-agarose conjugate was incubated with whole-cell extract of proximal or distal tubule cells $(1.5 \mathrm{ml})$ for $1 \mathrm{~h}$ at $4^{\circ} \mathrm{C}$. The beads with bound proteins were spun down by brief centrifugation, washed three times with the same lysis buffer at $4^{\circ} \mathrm{C}$. The proteins were then removed from gentamicinagarose in SDS sample buffer, boiled for $2 \mathrm{~min}$, and separated by protein gel electrophoresis $(8 \%$ gel) prior to Coomassie blue staining.

Mass spectrometry. Gentamicin-binding protein bands from KPT11 cells were excised from Coomassie-stained gels, washed twice for $30 \mathrm{~min}$ in $50 \mathrm{mM}$ ammonium bicarbonate $/ 50 \%(\mathrm{v} / \mathrm{v})$ acetonitrile, reduced in $10 \mathrm{mM}$ DTT/100 mM ammonium bicarbonate, alkylated in $55 \mathrm{mM}$ iodoacetamide/ $100 \mathrm{mM}$ ammonium bicarbonate, and dehydrated gels reswelled in digestion buffer containing $50 \mathrm{mM}$ ammonium bicarbonate $/ 5 \mathrm{mM} \mathrm{CaCl} / 12.5 \mathrm{ng} / \mu$ l sequencing grade modified trypsin (Promega, Madison, WI, USA). Following an overnight incubation at $37^{\circ} \mathrm{C}$, extracted peptides were identified by collection of MS/MS data using either a LTQ linear ion trap mass spectrometer (ThermoFisher, San Jose, CA, USA) or a Q-Star $X L$ hybrid quadrupole-TOF mass spectrometer (Applied Biosystems, Foster City, CA, USA). Peptides analyzed using the LTQ were separated using an Agilent 1100 series capillary LC system (Agilent Technologies Inc., Santa Clara, CA, USA) and $0.5 \times 250 \mathrm{~mm}$ Zorbax SB-C18 column (Agilent Technologies), and peptides analyzed by the Q-Star XL were separated using a nano LC system (Eksigent Technologies, Dublin, CA, USA) and $75 \mu \mathrm{m} \times 10 \mathrm{~cm}$ nanospray column filled with Astrosil C18 packing (Stellar Phases Inc., Langhorne, PA, USA). Proteins were identified by collection of data-dependent MS/MS spectra and matching of the $\mathrm{MS} / \mathrm{MS}$ spectra to a mouse subset of the Uniprot, version 15.4 database (containing 60784 entries), and specifying a static +57 mass for cysteines, and a differential mass of +16 for methionines using the program Sequest (version 27, rev. 12, Thermo Finnigan, San Jose, CA, USA). The database was also amended with an equal number of decoy sequence-reversed entries to independently assess the protein false discovery rate. Protein identification was then validated using the program Scaffold (version 2_04_00, Proteome Software Inc., Portland, OR, USA), which uses a probabilistic model to estimate the peptide and protein false discovery rate. ${ }^{36,37}$ Minimum peptide and protein probabilities were set at 80 and $99 \%$, respectively, with a minimum of five unique peptide matches per protein. Using these criteria, there were no matches to the decoy proteins with reversed sequences. Cytoskeleton-associated protein 4 (B2RRB4, also known as CLIMP-63) was identified by 24 unique peptides, tropomyosin $1 \alpha$ (Q564G1) by 27 unique peptides, caldesmon 1 (Q8VCQ8) by 36 unique peptides, actin by 8 unique peptides, and myosin-9 (Q8VDD5, also known as myosin IIA) by 20 unique peptides. Because of the sequence homology, the identified peptides from actin matched equally well to $\beta$ - and $\gamma$-actins (P60710 and P63260). Thus, the specific isoform(s) of actin were not distinguished. Similar methods and criteria were used for identification of 14-3-3 proteins as CLIMP-63-binding proteins. Two separate experiments identified 14-3-3 $\theta$ (experiment no. 1: six unique peptides, experiment no. 2: four unique peptides), 14-3-3 $\beta$ (experiment no. 1: four unique peptides,

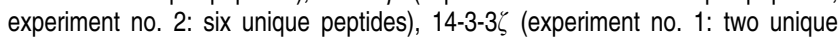

peptides, experiment no. 2: four unique peptides), and 14-3-3e (experiment no. 1: not identified, experiment no. 2: two unique peptides).

Western blotting. Proteins extracted from cells in lysis buffer were mixed with SDS sample buffer with or without DTT (final concentration of DTT at $0.6 \mathrm{M}$ ), resolved in a SDS-polyacrylamide gel, and transferred to PVDF membrane. After blocking and primary antibody incubation, horseradish peroxidase (HRP)conjugated secondary antibodies with chemiluminescence (Pierce, Rockford, IL, USA) were used to detect protein expression.

GTTR treatment and immunofluorescence. GTTR was generated and purified as described previously. ${ }^{10}$ All the following steps of incubation before fixation were performed under normal culture condition $\left(37^{\circ} \mathrm{C}, 5 \% \mathrm{CO}_{2}\right)$. Cells were preincubated in culture medium containing $2 \mathrm{mg} / \mathrm{ml} \mathrm{HRP} \mathrm{(Sigma)} \mathrm{for} 30 \mathrm{~min}$, and then washed twice and incubated in normal medium for 30 min before GTTR treatment. Cells were incubated with $5 \mu \mathrm{g} / \mathrm{ml}$ GTTR in medium for $1 \mathrm{~h}$ and washed with PBS three times before fixation with $4 \%$ of formaldehyde. To quench lysosomal fluorescence, fixed cells were incubated in diaminobenzidine solution (Vector Laboratories, Burlingame, CA, USA) for $5 \mathrm{~min}$, before permeabilization with $0.2 \%$ Triton X-100 for immunofluorescence. After blocking and primary antibody incubation, samples were incubated with AlexaFluor 488-conjugated secondary antibody (Invitrogen, Carlsbad, CA, USA) and protein expression was detected by confocal microscopy.

RT-PCR. Total RNA was extracted from mouse tissues or cells using RNeasy Mini Kit (Qiagen, Valencia, CA, USA), and cDNA was synthesized using SuperScript III First-Strand Synthesis System (Invitrogen). For mouse tissue comparison, cDNA was synthesized from FirstChoice Mouse Total RNA (Ambion, Austin, TX, USA). PCR was performed using the CDNA as template, AmpliTaq DNA polymerase (Applied Biosystems), and primers specific for mouse CLIMP-63 (5'CAGCGACTTGAAGGAGCTGAAG-3' and $5^{\prime}$-ACAACCTATCCAGGTCGTTCC G-3'), $\beta$-actin (5'-TGTTGCTCTAGACTTCGAGCAG- $3^{\prime}$ and $5^{\prime}$-ACCGATCCAC ACAGAGTACTTG-3') or GAPDH (5'-ATGTGTCCGTCGTGGATCTGAC- $3^{\prime}$ and 5'TACTCCTTGGAGGCCATGTAGG-3').

GST fusion protein generation and pull-down assays. The cytosolic and lumenal domains of human CLIMP-63 CDNA (IMAGE clone 6084968, ATCC, Manassas, VA, USA) were subcloned into PGEX5X-1 vector, by PCR amplification of each domain with following primers: for the cytosolic domain, $5^{\prime}-\mathrm{CC}$ CGAATTCATGCCCTCGGCCAAACAAAGG-3' and 5'-AAACTCGAGCCTGCCGA GCCTGCGCGAGCAG-3', and for the lumenal domain, $5^{\prime}$-CCCGAATTCCAC CACGTCCTGGAGGAGGTC- $3^{\prime}$ and $5^{\prime}$-GGGCTCGAGTTAGACCTTTTCGTGAAT CTTCTC- $3^{\prime}$. The resultant constructs were transformed into BL21 for bacterial protein expression. The GST fusions were generated and purified using BugBuster GST•Bind Purification Kit (Novagen, Gibbstown, NJ, USA). Gentamicin-agarose pull-down assay was performed using lysis buffer containing $150 \mathrm{mM} \mathrm{NaCl}, 20 \mathrm{mM}$ Tris (pH 7.5), $0.2 \%$ Triton X-100, $10 \%$ glycerol. For GST fusion protein pull-down assay, $5 \mu \mathrm{g}$ GST or GST fusion protein was bound to $30 \mu \mathrm{l}$ of $50 \%$ slurry of GST•Bind resin at $4^{\circ} \mathrm{C}$ for $2 \mathrm{~h}$ in GST•Bind Wash buffer. After washing for three times, cell lysate was mixed with the resin, and binding was performed at $4^{\circ} \mathrm{C}$ for $2 \mathrm{~h}$. Washing was performed with the lysis buffer four times after binding, and the proteins were removed from the resin in SDS sample buffer.

FLAG immunoprecipitation. Anti-FLAG M2 affinity gel (30 $\mu$ l; Sigma) was mixed with lysate from cells transfected with FLAG-tagged protein, and binding was performed at $4^{\circ} \mathrm{C}$ for $2 \mathrm{~h}$. After washing with Tris-buffered Saline for three times, bound proteins were eluted with $3 \times$ FLAG peptide (Sigma) at $4^{\circ} \mathrm{C}$ for $30 \mathrm{~min}$. For silver staining, SilverQuest Silver Staining Kit (Invitrogen) was used according to manufacturer's instruction.

Transfection. Two sets of siRNA for CLIMP-63 and scrambled control were designed and synthesized using Invitrogen's service. The sequences for CLIMP-63 siRNA are: no. $15^{\prime}$-CCAAGUCUAUCAAUGACAATT- $3^{\prime}$ and no. 2 $5^{\prime}$-GCAGAAUGAGAUUCUCAAATT-3'. For 14-3-3 siRNA, the sequences are:

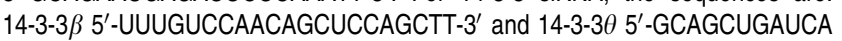
AGGACUAUTT- $3^{\prime}$. Control siRNA are scrambled sequences of the respective siRNA. Transfection of siRNA was performed using Lipofectamine RNAiMAX (Invitrogen). For expression plasmid transfection into 293T or COS-7 cells, human CLIMP-63 was subcloned into pcDNA3.1 vector. The point mutations were introduced by QuikChange Site-Directed Mutagenesis Kit (Stratagene, La Jolla, CA, 
USA) with primers: $5^{\prime}$-CTCGGCGTCCTCCTCGCGCAGGC-3' and $5^{\prime}$-GCCTG CGCGAGGAGGACGCCGAG-3' for C100S mutant, and $5^{\prime}$-CCTCGGCGTCC GCCTCGCGCAGGC-3' and 5'-GCCTGCGCGAGGCGGACGCCGAGG-3' for C100A mutant. The resultant plasmids were sequenced to confirm that there is no false mutation within the coding region of CLIMP-63. For 14-3-3 protein expression, human 14-3-3 $\beta$ and $\theta$ CDNA clones (cat. no. SC118005 and SC115879) were obtained from OriGene (Rockville, MD, USA), and subcloned into pFLAG-CMV2 vector, or pcDNA3.1 with addition of a Myc tag at the N-terminus. Plasmid transfection was carried out using LipofectAMINE 2000 (Invitrogen).

Cell viability measurements. Cell viability was determined by the reduction of 3-(4,5-dimethylthiazol-2-yl)-2,5-diphenyltetrazolium bromide (MTT), an indicator of mitochondrial dehydrogenase activity. Cells were plated at 10000 cells per well in a 96-well plate. After incubation overnight to allow cells to attach to the plate, cells were treated with gentamicin in culture medium for 3 days. For siRNA-transfected cells, cells were incubated for 3 days to ensure maximum knock down before 3-day gentamicin treatment. Cells were initially plated at 2500 cells per well to allow 6-day culturing (3-day transfection plus 3-day gentamicin treatment) without cellular overgrowth. There was no significant cellular detachment from plates by cell death during the 3-day transfection. After 3 days of gentamicin treatment, $20 \mu$ of $5 \mathrm{mg} / \mathrm{ml}$ of MTT solution was added to each well, and cells were incubated for $4 \mathrm{~h}$ at $37^{\circ} \mathrm{C}$ $5 \% \mathrm{CO}_{2}$. Culture medium was then replaced with $200 \mu \mathrm{lDMSO}$ in each well, and the optical density recorded at $540 \mathrm{~nm}$ with background subtraction at $660 \mathrm{~nm}$.

Caspase-3 assay. Cells were plated at $2 \times 10^{5}$ cells per $60-\mathrm{mm}$ dish. For KPT11 cells to be transfected with siRNA, cells were plated at $1.5 \times 10^{5}$ cells per dish. After incubation overnight, cells were treated with gentamicin in culture medium for 12 , 24, or $48 \mathrm{~h}$. Caspase-3 activity was determined by using CaspACE Assay System (Promega, Madison, WI, USA), according to the manufacturer's instructions.

TUNEL assay. After 3 days of siRNA transfection followed by 24-h gentamicin treatment, DNA strand breaks were labeled by TUNEL reaction by using In Situ Cell Detection Kit (Roche Applied Science, Indianapolis, IN, USA), and detected by confocal microscopy. Double-labeling with AlexaFluor 568-conjugated phalloidin was performed to visualize the filamentous actin cytoskeleton.

\section{Conflict of interest}

The authors declare no conflict of interest.

Acknowledgements. We thank Dr. Jack Rohrer for CLIMP-63 antibody, Dr. Federico Kalinec for HEI-OC1 and SV-k1 cells, and Dr. Stefan Heller for TRPV4 antibody. This work was supported by the NIH NIDCD grant R03 DC009501 (TK), R01 DC04555 (PSS), and P30 grants DC05983, EY10572, and CA069533.

1. Forge A, Schacht J. Aminoglycoside antibiotics. Audiol Neurootol 2000; 5: 3-22.

2. Edson RS, Terrell CL. The aminoglycosides. Mayo Clin Proc 1999; 74: 519-528.

3. Grohskopf LA, Huskins WC, Sinkowitz-Cochran RL, Levine GL, Goldmann DA, Jarvis WR Use of antimicrobial agents in United States neonatal and pediatric intensive care patients. Pediatr Infect Dis J 2005; 24: 766-773.

4. Twiss J, Byrnes $C$, Johnson R, Holland D. Nebulised gentamicin-suitable for childhood bronchiectasis. Int J Pharm 2005; 295: 113-119.

5. Tulkens PM. Nephrotoxicity of aminoglycoside antibiotics. Toxicol Lett 1989; 46: 107-123.

6. Lowenheim H, Furness DN, Kil J, Zinn C, Gultig K, Fero ML et al. Gene disruption of p27(Kip1) allows cell proliferation in the postnatal and adult organ of corti. Proc Natl Acad Sci USA 1999; 96: 4084-4088.

7. Hashino E, Shero M, Salvi RJ. Lysosomal targeting and accumulation of aminoglycoside antibiotics in sensory hair cells. Brain Res 1997; 777: 75-85.

8. Sandoval RM, Dunn KW, Molitoris BA. Gentamicin traffics rapidly and directly to the Golgi complex in LLC-PK(1) cells. Am J Physiol Renal Physiol 2000; 279: F884-F890.

9. Sandoval RM, Molitoris BA. Gentamicin traffics retrograde through the secretory pathway and is released in the cytosol via the endoplasmic reticulum. Am J Physiol Renal Physio 2004; 286: F617-F624

10. Myrdal SE, Johnson KC, Steyger PS. Cytoplasmic and intra-nuclear binding of gentamicin does not require endocytosis. Hear Res 2005; 204: 156-169.

11. Marcotti W, van Netten SM, Kros CJ. The aminoglycoside antibiotic dihydrostreptomycin rapidly enters mouse outer hair cells through the mechano-electrical transducer channels. J Physiol 2005; 567(Part 2): 505-521.
12. Karasawa T, Wang Q, Fu Y, Cohen DM, Steyger PS. TRPV4 enhances the cellular uptake of aminoglycoside antibiotics. J Cell Sci 2008; 121(Part 17): 2871-2879.

13. Lee JE, Nakagawa T, Kim TS, Iguchi F, Endo T, Kita T et al. Signaling pathway for apoptosis of vestibular hair cells of mice due to aminoglycosides. Acta Otolaryngol 2004; 551: 69-74.

14. Hirose K, Hockenbery DM, Rubel EW. Reactive oxygen species in chick hair cells after gentamicin exposure in vitro. Hear Res 1997; 104: 1-14.

15. Pirvola U, Xing-Qun L, Virkkala J, Saarma M, Murakata C, Camoratto AM et al. Rescue of hearing, auditory hair cells, and neurons by CEP-1347/KT7515, an inhibitor of c-Jun N-terminal kinase activation. J Neurosci 2000; 20: 43-50.

16. Sha SH, Schacht J. Antioxidants attenuate gentamicin-induced free radical formation in vitro and ototoxicity in vivo: D-methionine is a potential protectant. Hear Res 2000; 142: 34-40.

17. Jiang $\mathrm{H}$, Sha SH, Forge A, Schacht J. Caspase-independent pathways of hair cell death induced by kanamycin in vivo. Cell Death Differ 2006; 13: 20-30.

18. Klopfenstein DR, Kappeler F, Hauri HP. A novel direct interaction of endoplasmic reticulum with microtubules. Embo J 1998; 17: 6168-6177.

19. Klopfenstein DR, Klumperman J, Lustig A, Kammerer RA, Oorschot V, Hauri HP. Subdomain-specific localization of CLIMP-63 (p63) in the endoplasmic reticulum is mediated by its luminal alpha-helical segment. J Cell Biol 2001; 153: 1287-1300.

20. Tian W, Salanova M, Xu H, Lindsley JN, Oyama TT, Anderson S et al. Renal expression of osmotically responsive cation channel TRPV4 is restricted to water-impermeant nephron segments. Am J Physiol Renal Physiol 2004; 287: F17-F24.

21. Vedrenne C, Hauri HP. Morphogenesis of the endoplasmic reticulum: beyond active membrane expansion. Traffic (Copenhagen, Denmark) 2006; 7: 639-646.

22. Gesek FA, Friedman PA. Mechanism of calcium transport stimulated by chlorothiazide in mouse distal convoluted tubule cells. J Clin Invest 1992; 90: 429-438.

23. Horibe T, Matsui H, Tanaka M, Nagai H, Yamaguchi $Y$, Kato $\mathrm{K}$ et al. Gentamicin binds to the lectin site of calreticulin and inhibits its chaperone activity. Biochem Biophys Res Commun 2004; 323: 281-287.

24. Schweizer A, Ericsson M, Bachi T, Griffiths G, Hauri HP. Characterization of a novel $63 \mathrm{kDa}$ membrane protein. Implications for the organization of the ER-to-Golgi pathway. J Cell Sci 1993; 104(Part 3): 671-683.

25. Espreafico EM, Coling DE, Tsakraklides V, Krogh K, Wolenski JS, Kalinec G et al. Localization of myosin-V in the centrosome. Proc Natl Acad Sci USA 1998; 95: 8636-8641.

26. Kalinec GM, Webster $P$, Lim DJ, Kalinec F. A cochlear cell line as an in vitro system for drug ototoxicity screening. Audiol Neurootol 2003; 8: 177-189.

27. Zhang J, Planey SL, Ceballos C, Stevens Jr SM, Keay SK, Zacharias DA. Identification of CKAP 4/p63 as a major substrate of the palmitoyl acyltransferase DHHC2, a putative tumor suppressor, using a novel proteomics method. Mol Cell Proteomics 2008; 7: 1378-1388.

28. Planey SL, Keay SK, Zhang CO, Zacharias DA. Palmitoylation of cytoskeleton associated protein 4 by DHHC2 regulates antiproliferative factor-mediated signaling. Mol Biol Cell 2009; 20: 1454-1463.

29. Morrison DK. The 14-3-3 proteins: integrators of diverse signaling cues that impact cell fate and cancer development. Trends Cell Biol 2009; 19: 16-23.

30. Miyazaki T, Sagawa R, Honma T, Noguchi S, Harada T, Komatsuda A et al. 73-kDa molecular chaperone HSP73 is a direct target of antibiotic gentamicin. J Biol Chem 2004; 279: $17295-17300$.

31. Muller E, Neuhofer W, Ohno A, Rucker S, Thurau K, Beck FX. Heat shock proteins HSP25, HSP60, HSP72, HSP73 in isoosmotic cortex and hyperosmotic medulla of rat kidney. Pflugers Arch 1996; 431: 608-617.

32. Myrdal SE, Steyger PS. TRPV1 regulators mediate gentamicin penetration of cultured kidney cells. Hear Res 2005; 204: 170-182.

33. Liang X, Butterworth MB, Peters KW, Walker WH, Frizzell RA. An obligatory heterodimer of 14-3-3beta and 14-3-3epsilon is required for aldosterone regulation of the epithelial sodium channel. J Biol Chem 2008; 283: 27418-27425.

34. Nakamura T, Hayashi T, Mimori-Kiyosue Y, Sakaue F, Matsuura K, lemura S et al. The PX-RICS-14-3-3zeta/theta complex couples N-cadherin-beta-catenin with dyneindynactin to mediate its export from the endoplasmic reticulum. J Biol Chem 2010; 285: 16145-16154.

35. Wesierska-Gadek J, Hajek SB, Sarg B, Wandl S, Walzi E, Lindner H. Pleiotropic effects of selective CDK inhibitors on human normal and cancer cells. Biochem Pharmacol 2008; 76: 1503-1514.

36. Keller A, Nesvizhskii Al, Kolker E, Aebersold R. Empirical statistical model to estimate the accuracy of peptide identifications made by MS/MS and database search. Anal Chem 2002; 74: 5383-5392.

37. Nesvizhskii Al, Keller A, Kolker E, Aebersold R. A statistical model for identifying proteins by tandem mass spectrometry. Anal Chem 2003; 75: 4646-4658.

Cell Death and Disease is an open-access journal published by Nature Publishing Group. This work is licensed under the Creative Commons Attribution-Noncommercial-No Derivative Works 3.0 Unported License. To view a copy of this license, visit http://creativecommons.org/licenses/by-nc-nd/3.0/ 